\title{
Lower bounds in real Schubert calculus
}

\section{Nickolas Hein}

Department of Mathematics,University of Nebraska at Kearney

Kearney 68849 Nebraska, USA

E-mail address: heinnj@unk.edu

URL: http://www. unk.edu/academics/math/faculty/About_Nickolas_Hein/

\section{Christopher J. Hillar}

Redwood Center for Theoretical Neuroscience

University of California

Berkeley 94720 California, USA

E-mail address: chillar@msri.org

URL: http://www.msri.org/people/members/chillar/

\section{Frank Sottile}

Department of Mathematics

Texas A\&M University

College Station 77843 Texas, USA

E-mail address: sottile@math.tamu.edu

$U R L:$ http://www.math.tamu.edu/ sottile

\begin{abstract}
We describe a large-scale computational experiment studying structure in the numbers of real solutions to osculating instances of Schubert problems. This investigation uncovered Schubert problems whose computed numbers of real solutions variously exhibit nontrivial upper bounds, lower bounds, gaps, and a congruence modulo four. We present a family of Schubert problems, one in each Grassmannian, and prove that their real osculating instances have the observed lower bounds and gaps.
\end{abstract}

2010 Mathematics Subject Classification. 14N15, 14 P99.

Key words: Schubert calculus, Shapiro Conjecture, Lower bounds.

Research of Sottile and Hein supported in part by NSF grants DMS-0079536, DMS1001615, and DMS-0922866. Hillar supported by NSF grant IIS-1219212.

The work represents part of Hein's 2013 Ph.D. thesis from Texas A\&M University.

Based upon work supported by the NSF under Grant No. 0932078000 while Sottile was in residence at the Mathematical Science Research Institute in Berkeley, California, during the winter semester of 2013. 


\section{Introduction}

A remarkable recent story in mathematics was the proof of the Shapiro Conjecture (in real algebraic geometry) by Mukhin, Tarasov, and Varchenko [20] using methods from integrable systems. Its simplest form involves the Wronski map, which sends a $k$-dimensional complex linear subspace of univariate polynomials of degree $n-1$ to its Wronskian, a polynomial of degree $k(n-k)$. In this context, the Mukhin-Tarasov-Varchenko Theorem states that if a polynomial $w(t)$ of degree $k(n-k)$ has all of its roots real, then every $k$-plane of polynomials with Wronskian $w(t)$ is real (i.e., has a basis of real polynomials).

The Wronskian is a map from a Grassmannian to a projective space, both of dimension $k(n-k)$. Eremenko and Gabrielov [6] considered the real Wronski map that sends the real Grassmannian to real projective space, computing its topological degree (actually the degree of a lift to oriented double covers). This topological degree is strictly positive when $n$ is odd, so that for $n$ odd, there are always real $k$-planes of polynomials with given real Wronskian, proving a weak version of the Shapiro Conjecture.

The full Shapiro Conjecture went far beyond the reality of the Wronski map. More generally it concerned intersections of Schubert varieties given by flags osculating a rational normal curve (osculating instances of Schubert problems), positing that if the osculating points were all real, then all of the points of intersection were also real. When the Schubert varieties are all hypersurfaces, the conjecture asserted that the fibers of the Wronski map over polynomials with only real roots contained only real subspaces. Initially considered too strong to be true, the Shapiro Conjecture came to be accepted due to significant computer experimentation $[27,32]$ and partial results $[8,26]$.

Fibers of the Wronski map over a polynomial $w(t)$ with distinct roots are intersections of hypersurface Schubert varieties given by flags osculating the rational normal curve at the roots of $w(t)$. When $w(t)$ is real, its roots form a real variety (stable under complex conjugation) and the corresponding intersection of Schubert varieties is also real. Eremenko and Gabrielov's topological degree is a lower bound for the number of real points in that real intersection. In related work, Azar and Gabrielov [1] proved a lower bound for the number of real rational functions of degree $d$ with $2 d-3$ real critical points and two real points where the function values coincide. (This is a lower bound for a family of Schubert problems on a flag manifold given by osculating flags and was motivated by data from the experiment [24].) These results suggested the possibility of lower bounds for the number of real points in an intersection of Schubert varieties given by flags osculating the rational normal curve, when the intersection is a real variety. 
A preliminary investigation [13] confirmed this possibility and uncovered other structures in the numbers of real solutions, including upper bounds, lower bounds, gaps, and a congruence modulo four, in different families of Schubert problems. Those data led to three papers $[23,16,17]$ which proved some of the observed structure.

We describe the design, execution, and some results of a large-scale computer experiment [15] to study such real osculating instances of Schubert problems. This study investigated over 344 million instances of $756 \mathrm{Schu-}$ bert problems, and it used over 549 gigahertz-years of computing. The topological lower bounds of Eremenko and Gabrielov [6] apply to variants of the Wronski map and were extended by Soprunova and Sottile [25] to give topological lower bounds to osculating Schubert problems where at most two Schubert varieties were not hypersurfaces. We studied 273 such osculating Schubert problems and observed that these topological lower bounds were sharp for all except six of them.

Four of these six continue to defy explanation. For the remaining two, the lack of sharpness is due to a congruence modulo four observed in both [13] and [15] for certain symmetric Schubert problems. This congruence has since been established by Hein and Sottile in collaboration with Zelenko. They first [16] treated osculating Schubert problems and established a weak form of the congruence. Later, they showed that many symmetric Schubert problems in a Grassmannian given by symplectic flags have a congruence modulo four on their number of real solutions [17].

For example, Table 1 summarizes the computation for two Schubert problems in $\operatorname{Gr}(4,8)$, each with twelve solutions. Together these used 202

TABLE 1. Frequency of observed number of real solutions

\begin{tabular}{|c|c|c|c|c|c|c|c|c|}
\hline \multirow{2}{*}{ Problem } & \multicolumn{7}{|c|}{ Number of Real Solutions } & \multirow{2}{*}{ Total } \\
\hline & 0 & 2 & 4 & 6 & 8 & 10 & 12 & \\
\hline$\boxplus^{3} \cdot \boxplus \cdot \square$ & 81912 & & 88738 & & 7086 & & 222264 & 400000 \\
\hline 田. 田 ${ }^{2} \cdot \square^{3}$ & 214375 & & 231018 & & 61600 & & 293007 & 800000 \\
\hline
\end{tabular}

gigahertz-days of computing. The columns are the number of observed instances with a given number of real solutions. We only list even numbers, for the number of real solutions is always congruent modulo two to the number of complex solutions. Empty cells indicate that no instances were observed with that number of real solutions. Notice that the partitions encoding the Schubert problems are symmetric, and that the observed numbers of real solutions satisfy an additional congruence modulo four. This congruence occurs for a symmetric Schubert problem in $\operatorname{Gr}(k, 2 k)$ when the 
sum of the lengths of the diagonals of its partitions is at least $k+4$. This sum is eight for both problems of Table 1.

Five problems studied exhibited lower bounds and other structures in their observed numbers of real solutions. These problems form members of a family of Schubert problems, one in each Grassmannian, which we prove have this unusual structure on numbers of real solutions. We shall show that real solutions to an osculating instance correspond to real factorizations of an associated polynomial, explaining the observed structures.

This paper is organized as follows. In Section 1 we provide background on the Schubert calculus, the history of the Shapiro Conjecture, and the work of Eremenko and Gabrielov on topological lower bounds. We then describe the setup, execution, and some of the observations resulting from the experimental project in Section 2. In Section 3, we explain the lower bounds and gaps coming from a family of Schubert problems whose determination we reduce to factoring certain polynomials. In Section 4, we conclude with a discussion of some frequency tables exhibiting interesting structure.

\section{Background}

We first establish our notation and definitions regarding the osculating Schubert calculus, give some additional history of the Shapiro Conjecture, and finally discuss the topological lower bounds of Eremenko-Gabrielov and Soprunova-Sottile.

1.1. Osculating Schubert calculus. Let $k<n$ be positive integers. The Grassmannian $\operatorname{Gr}(k, n)$ (or $\operatorname{Gr}\left(k, \mathbb{C}^{n}\right)$ ) is the set of all $k$-dimensional linear subspaces ( $k$-planes) of $\mathbb{C}^{n}$, which is a complex manifold of dimension $k(n-k)$. Complex conjugation on $\mathbb{C}^{n}$ induces a conjugation on $\operatorname{Gr}(k, n)$. The points of $\operatorname{Gr}(k, n)$ fixed by conjugation are its real points, and they form the Grassmannian $\operatorname{Gr}\left(k, \mathbb{R}^{n}\right)$ of $k$-planes in $\mathbb{R}^{n}$.

The Grassmannian has distinguished Schubert varieties, which are given by the discrete data of a partition and the continuous data of a flag. A partition is a weakly decreasing sequence $\lambda: n-k \geq \lambda_{1} \geq \cdots \geq \lambda_{k} \geq 0$ of integers and a flag is a filtration of $\mathbb{C}^{n}$ :

$$
F_{\bullet}: F_{1} \subsetneq F_{2} \subsetneq \cdots \subsetneq F_{n}=\mathbb{C}^{n},
$$

where $\operatorname{dim} F_{i}=i$. The flag $F_{\bullet}$ is real if $\overline{F_{i}}=F_{i}$ for all $i$, so that it is the complexification of a flag in $\mathbb{R}^{n}$. Given a partition $\lambda$ and a flag $F_{\bullet}$, the associated Schubert variety is

$$
X_{\lambda} F_{\bullet}:=\left\{H \in \operatorname{Gr}(k, n) \mid \operatorname{dim} H \cap F_{n-k+i-\lambda_{i}} \geq i \text { for } i=1, \ldots, k\right\} .
$$


This is an irreducible subvariety of the Grassmannian of codimension $|\lambda|:=$ $\lambda_{1}+\cdots+\lambda_{k}$. From the definition, we see that $\overline{X_{\lambda} F_{\bullet}}=X_{\lambda} \overline{F_{\bullet}}$. tion

A list $\boldsymbol{\lambda}=\left(\lambda^{1}, \ldots, \lambda^{m}\right)$ of partitions which satisfies the numerical condi-

$$
\left|\lambda^{1}\right|+\left|\lambda^{2}\right|+\cdots+\left|\lambda^{m}\right|=k(n-k)
$$

is a Schubert problem. Given a Schubert problem $\boldsymbol{\lambda}$ and general flags $F_{\bullet}^{1}, \ldots, F_{\bullet}^{m}$, Kleiman's Transversality Theorem [19] implies that the intersection

$$
X_{\lambda^{1}} F_{\bullet}^{1} \cap X_{\lambda^{2}} F_{\bullet}^{2} \cap \cdots \cap X_{\lambda^{m}} F_{\bullet}^{m}
$$

is generically transverse. The numerical condition (1.2) implies that it is zero-dimensional (or empty) and therefore consists of finitely many points. The number of points does not depend upon the choice of general flags and may be computed using algorithms from the Schubert calculus [10]. The intersection (1.3) is an instance of the Schubert problem $\boldsymbol{\lambda}$ and its points are the solutions to this instance.

We will not be concerned with general instances of Schubert problems but rather with instances given by flags that osculate a common rational normal curve. Let $\gamma: \mathbb{C} \rightarrow \mathbb{C}^{n}$ be the following parameterized rational normal curve

$$
\gamma(t):=\left(1, t, \frac{t^{2}}{2}, \frac{t^{3}}{3 !}, \ldots, \frac{t^{n-1}}{(n-1) !}\right) .
$$

(This choice of $\gamma$ is no restriction as all rational normal curves are projectively equivalent.) For each $t \in \mathbb{C}$, the osculating flag $F_{\bullet}(t)$ has as its $i$-dimensional subspace the $i$-plane $F_{i}(t)$ osculating the curve $\gamma$ at $\gamma(t)$ :

$$
\begin{aligned}
F_{i}(t) & :=\operatorname{span}\left\{\gamma(t), \gamma^{\prime}(t), \ldots, \gamma^{(i-1)}(t)\right\} \\
& =\operatorname{row} \text { space }\left(\frac{t^{b-a}}{(b-a) !}\right)_{\substack{a=1, \ldots, i \\
b=a, \ldots, n}} .
\end{aligned}
$$

(The remaining entries in this matrix are zero.)

An osculating instance of a Schubert problem $\boldsymbol{\lambda}$ is one given by osculating flags,

$$
X_{\lambda^{1}} F_{\bullet}\left(t_{1}\right) \cap X_{\lambda^{2}} F_{\bullet}\left(t_{2}\right) \cap \cdots \cap X_{\lambda^{m}} F_{\bullet}\left(t_{m}\right) .
$$

Here, $t_{1}, \ldots, t_{m}$ are distinct points of $\mathbb{P}^{1}$. Osculating flags are not general for intersections of Schubert varieties as demonstrated in $[24, \S 2.3 .6]$, so Kleiman's Theorem does not imply that the intersection (1.6) is transverse. However, Eisenbud and Harris [5] noted that if $H \in X_{\lambda} F_{\bullet}\left(t_{0}\right)$ then its Wronskian $w_{H}(t)$ vanishes to order $|\lambda|$ at $t=t_{0}$. As the Wronskian (a form on $\left.\mathbb{P}^{1}\right)$ has degree $k(n-k)$, they deduced that (1.6) is at most zerodimensional. Later, Mukhin, Tarasov, and Varchenko [21] showed that the intersection is transverse when $t_{1}, \ldots, t_{m}$ are real (and therefore also when they are general). 
Eisenbud and Harris also noted that if $t_{0}$ is a root of order $\ell$ of the Wronskian $w_{H}(t)$ of $H$, then there is a unique partition $\lambda$ with $|\lambda|=\ell$ such that $H \in X_{\lambda} F_{\bullet}\left(t_{0}\right)$. This implies the following partial converse to Schubert problems.

Proposition 1. For each $H \in \operatorname{Gr}(k, n)$, there is a unique Schubert problem $\boldsymbol{\lambda}$ and unique points $t_{1}, \ldots, t_{m} \in \mathbb{P}^{1}$ for which $H$ lies in the intersection (1.6).

To simplify notation, we henceforth write $X_{\lambda}(t)$ for the Schubert variety $X_{\lambda} F_{\bullet}(t)$.

As $\overline{\gamma^{(i)}(t)}=\gamma^{(i)}(\bar{t})$, we have $\overline{F_{\bullet}(t)}=F_{\bullet}(\bar{t})$, and therefore $\overline{X_{\lambda}(t)}=X_{\lambda}(\bar{t})$. A consequence of these observations and Proposition 1 is the following corollary.

Corollary 2. Let $\boldsymbol{\lambda}=\left(\lambda^{1}, \ldots, \lambda^{m}\right)$ be a Schubert problem and $t_{1}, \ldots, t_{m} \in$ $\mathbb{P}^{1}$ be distinct. The instance

$$
X_{\lambda^{1}}\left(t_{1}\right) \cap X_{\lambda^{2}}\left(t_{2}\right) \cap \cdots \cap X_{\lambda^{m}}\left(t_{m}\right)
$$

of the Schubert problem $\boldsymbol{\lambda}$ is a real variety if and only if for each $i=$ $1, \ldots, m$ there exists $1 \leq j \leq m$ such that $\lambda_{i}=\lambda_{j}$ and $t_{i}=\bar{t}_{j}$.

Corollary 2 asserts that the obviously sufficient condition for an osculating instance of a Schubert problem to be a real variety, namely that each complex conjugate pair of osculation points have the same Schubert condition, is in fact necessary.

1.2. The Shapiro Conjecture and its generalizations. One motivation for studying real osculating instances of Schubert problems is the conjecture of Shapiro and Shapiro, which was given two different proofs by Mukhin, Tarasov, and Varchenko.

Theorem 3 ([20,21]). Given any osculating instance of a Schubert problem

$$
X_{\lambda^{1}}\left(t_{1}\right) \cap X_{\lambda^{2}}\left(t_{2}\right) \cap \cdots \cap X_{\lambda^{m}}\left(t_{m}\right),
$$

in which $t_{1}, \ldots, t_{m} \in \mathbb{R P}^{1}$, the intersection is transverse with all of its points real.

The Shapiro Conjecture was made by the brothers Boris and Michael Shapiro in 1993, and popularized through significant computer experimentation and partial results $[27,32]$. An asymptotic version (where the points $t_{i}$ are sufficiently clustered and all except two of the $\lambda^{i}$ consist of one part) was proven in [26]. The first breakthrough was given by Eremenko and Gabrielov [8] who used complex analysis to prove it when $\min \{k, n-k\}=2$. In this case it is equivalent to the statement that a rational function whose 
critical points lie on a circle in $\mathbb{P}^{1}$ maps that circle to a circle. Later, Mukhin, Tarasov, and Varchenko proved the full conjecture [20, 21] using methods from mathematical physics.

While the Shapiro Conjecture may be formulated in any flag manifold, it is false in general (except for the orthogonal Grassmannian [22]). Significant experimental work has uncovered the limits of its validity, as well as generalizations and extensions that are likely true [11, 14, 24], and has led to a proof of one generalization (the Monotone Conjecture) in a special case [9]. For a complete account, see [28] or [29, Chs. 9-14].

1.3. Topological lower bounds. While studying the Shapiro Conjecture, Eremenko and Gabrielov looked at real osculating instances of the form

$$
X_{\square}\left(t_{1}\right) \cap X_{\square}\left(t_{2}\right) \cap \cdots \cap X_{\square}\left(t_{m}\right) \cap X_{\lambda}(\infty),
$$

where $m+|\lambda|=k(n-k)$ and $\left\{t_{1}, \ldots, t_{m}\right\}$ is a real set in that $\left\{t_{1}, \ldots, t_{m}\right\}=$ $\left\{\overline{t_{1}}, \ldots, \overline{t_{m}}\right\} \subset \mathbb{P}^{1}$, equivalently, $w(t):=\prod_{i}\left(t-t_{i}\right)$ is a real polynomial. The points in (1.7) are the fiber of the Wronski map over the real polynomial $w(t)$ restricted to the Schubert variety $X_{\lambda}(\infty)$. Eremenko and Gabrielov [6] gave a formula for the topological degree of this Wronski map restricted to the real points of $X_{\lambda}(\infty)$ (and lifted to an oriented double cover). This topological degree is a topological lower bound on the number of real points in the intersection (1.7). This follows from the formula for the topological degree of a map $f: X \rightarrow Y$ between oriented manifolds,

$$
\operatorname{deg} f=\sum_{x \in f^{-1}(y)} \operatorname{sign}\left(d f_{x}\right),
$$

where $y \in Y$ is a regular value of $f$ and $\operatorname{sign}\left(d f_{x}\right)$ is 1 if the orientation of $T_{y} Y$ given by the differential $d f_{x}\left(T_{x} X\right)$ agrees with its orientation from $Y$, and -1 if the orientations do not agree.

This was generalized by Soprunova and Sottile [25, Th. 6.4] to intersections of the form

$$
X_{\mu}(0) \cap X_{\square}\left(t_{1}\right) \cap X_{\square}\left(t_{2}\right) \cap \cdots \cap X_{\square}\left(t_{m}\right) \cap X_{\lambda}(\infty),
$$

where $m+|\lambda|+|\mu|=k(n-k)$ and $\left\{t_{1}, \ldots, t_{m}\right\} \subset \mathbb{C}^{*}$ is a real set. This intersection is again a fiber of the Wronski map restricted to $X_{\mu}(0) \cap X_{\lambda}(\infty)$ (and lifted to an oriented double cover). They expressed the topological degree in terms of sign-imbalance.

A partition $\lambda$ is represented by its Young diagram, which is a left-justified array of boxes with $\lambda_{i}$ boxes in row $i$. When $\mu \subset \lambda$, we have the skew partition $\lambda / \mu$, which is the set-theoretic difference $\lambda \backslash \mu$ of their diagrams. For example, if

$$
\lambda=\boxplus \text { and } \quad \mu=\boxplus \text { then } \quad \lambda / \mu=\boxplus \text {. }
$$


Given $\lambda$, let $\lambda^{c}$ be the partition $n-k-\lambda_{k} \geq \cdots \geq n-k-\lambda_{1}$, the difference between the $k \times(n-k)$ rectangle and $\lambda$. For example, if $k=3, n=7$, and $\lambda=(3,0,0)$, then $\lambda^{c}=(4,4,1)$. A Young tableau of shape $\lambda / \mu$ is a filling of the boxes in $\lambda / \mu$ with the consecutive integers $1,2, \ldots,|\lambda|-|\mu|$ which increases across each row and down each column. The standard filling is the tableau whose numbers are in reading order. Here are four tableaux of shape $(4,4,1) /(1)$. The first has the standard filling.

\begin{tabular}{|c|c|c|c|}
\hline \begin{tabular}{|l|l|l|}
1 & 2 & 3 \\
\end{tabular} & \begin{tabular}{|l|l|l|}
3 & 5 & 7 \\
\end{tabular} & \begin{tabular}{|l|l|l|}
2 & 3 & 5 \\
\end{tabular} & \begin{tabular}{|l|l|l|}
1 & 3 & 6 \\
\end{tabular} \\
\hline \begin{tabular}{|l|l|l|l}
4 & 5 & 6 & 7 \\
\end{tabular} & \begin{tabular}{|l|l|l|l|}
1 & 4 & 6 & 8 \\
\end{tabular} & \begin{tabular}{|l|l|l|l|}
1 & 4 & 6 & 7 \\
\end{tabular} & \begin{tabular}{|l|l|l|l|}
2 & 4 & 5 & 8 \\
\end{tabular} \\
\hline \begin{tabular}{|l|l|}
8 & \\
\end{tabular} & \begin{tabular}{|l|ll}
2 & \\
\end{tabular} & \begin{tabular}{|l|l|}
8 & \\
\end{tabular} & \begin{tabular}{|l|ll}
7 & & \\
\end{tabular} \\
\hline
\end{tabular}

Let $Y(\lambda / \mu)$ be the set of Young tableaux of shape $\lambda / \mu$. Each tableau $T$ has a parity, $\operatorname{sign}(T) \in\{ \pm 1\}$, which is the sign of the permutation mapping the standard filling to $T$. The sign-imbalance of $\lambda / \mu$ is

$$
\sigma(\lambda / \mu):=\left|\sum_{T \in Y(\lambda / \mu)} \operatorname{sign}(T)\right|
$$

Algorithms in the Schubert calculus [10] imply that the number of complex points in the intersection (1.8) is the number $\left|Y\left(\lambda^{c} / \mu\right)\right|$ of tableaux of shape $\lambda^{c} / \mu$. Soprunova and Sottile show that the topological degree of the appropriate Wronski map is the sign-imbalance of $\lambda^{c} / \mu$. We deduce the following proposition.

Proposition $4([6,25])$. If $\left\{t_{1}, \ldots, t_{m}\right\} \subset \mathbb{C}^{*}$ is a real set, then the number of real points in the intersection (1.8) is at least the sign-imbalance $\sigma\left(\lambda^{c} / \mu\right)$ of $\lambda^{c} / \mu$.

When $\lambda=\mu=\emptyset$, Eremenko and Gabrielov gave a closed formula for this topological lower bound, which showed that it is strictly positive when $n$ is odd and zero when $n$ is even [6]. Later they showed that if both $n$ and $k$ are even, there is an intersection (1.7) with no real points [7], showing in these cases that the topological lower bound is sharp. Investigating when the topological lower bounds of Proposition 4 are sharp and when they are not was a focus of the experiment.

\section{Experimental project}

We describe a large computational experiment to study structure in the number of real solutions to real instances of osculating Schubert problems. The data for this experiment kept track of which pairs of the osculating flags were complex conjugate, as preliminary computations and the Mukhin-Tarasov-Varchenko Theorem showed that this affected the numbers of real solutions. The computations were carried out symbolically in exact arithmetic, with real osculating instances of Schubert problems being 
formulated as systems of polynomial equations. We sketch the execution of the experiment and then close with a discussion of some of the data gathered, which is available to browse online [15].

2.1. Osculation type. We have expressed Schubert problems $\boldsymbol{\lambda}$ as lists of partitions. Also useful and more compact is multiplicative notation. For example, the Schubert problem $\boldsymbol{\lambda}=(\boxplus, \Xi, \square, \square, \square)$ in $\operatorname{Gr}(3,6)$ with six solutions is written multiplicatively as $\Xi^{2} \cdot \square^{3}$ or as $\Xi^{2} \cdot \square^{3}=6$, when we wish to give its number of solutions.

The topological lower bound for the Schubert problem $\boxplus \cdot \square^{5}=6$ in $\operatorname{Gr}(3,6)$ is the sign imbalance $\sigma\left(\mathbb{\boxplus}^{c}\right)=\sigma\left(\mathbb{\boxplus}^{\boxplus}\right)=2$. Thus, if the instance

$$
X_{\boxplus}(0) \cap X_{\square}\left(t_{1}\right) \cap \cdots \cap X_{\square}\left(t_{5}\right)
$$

of the Schubert problem $\boxplus \cdot \square^{5}=6$ has $\left\{\overline{t_{1}}, \ldots, \overline{t_{5}}\right\}=\left\{t_{1}, \ldots, t_{5}\right\}$, then $(2.1)$ contains at least two real points. If $t_{1}, \ldots, t_{5} \in \mathbb{R P}^{1}$ then all six points in (2.1) are real by the Mukhin-Tarasov-Varchenko Theorem. This illustrates that the lower bound on the number of real solutions to an osculating instance of a Schubert problem is sensitive to the number of real osculation points. Given a Schubert problem $\left(\lambda^{1}\right)^{a_{1}} \cdots\left(\lambda^{m}\right)^{a_{m}}$ and a corresponding real osculating instance $X$, the osculation type $r$ of $X$ is the list $r=\left(r_{\lambda^{1}}, \ldots, r_{\lambda^{m}}\right)$ where $r_{\lambda^{i}}$ is the number of Schubert varieties of the form $X_{\lambda^{i}}(t)$ containing $X$ with $t$ real. Since $X$ is real, we have $r_{\lambda^{i}} \equiv a_{i} \bmod 2$ for each $i$.

Table 2 is from the experiment. It records how often a given number of real solutions was observed for a given osculation type in 400000 random real instances of the Schubert problem $\square \square \square \cdot \square^{7}=6$. In every

TABLE 2. Frequency table for $\square \Pi \square \cdot \square^{7}=6$ in $\operatorname{Gr}(2,8)$

\begin{tabular}{|c||r|r|r|r||r|}
\hline \multicolumn{1}{|c||}{$r_{\square}$} & \multicolumn{3}{c||}{ Number of Real Solutions } & \multirow{2}{*}{ Total } \\
\cline { 2 - 5 } & 0 & 2 & 4 & 6 & \\
\hline \hline 7 & & & & 100000 & 100000 \\
\hline 5 & & & 77134 & 22866 & 100000 \\
\hline 3 & & 47138 & 47044 & 5818 & 100000 \\
\hline 1 & 8964 & 67581 & 22105 & 1350 & 100000 \\
\hline
\end{tabular}

computed instance when $r_{\square}=7$, all six solutions were real, agreeing with the Mukhin-Tarasov-Varchenko Theorem. The nonzero entry 8964 in the bottom row with $r_{\square}=1$ indicates the sharpness of the topological lower bound $\sigma\left(\square \square \square^{c}\right)=0$ for $\square \square \square \cdot \square^{7}$. The table suggests the lower 
bound of $r_{\square}-1$ for the number of real solutions to an instance of this Schubert problem, which we prove in Section 3. Studying this Schubert problem used 1.8 gigahertz-days of computing.

2.2. Schubert problems in local coordinates. The fundamental fact that underlies this experiment is that we may represent Schubert problems on a computer through systems of equations, from which we may extract the number of real solutions. We explain how to formulate Schubert problems as systems of equations.

A point in the Grassmannian $\operatorname{Gr}(k, n)$ is the row space of a matrix $M \in$ Mat $_{k \times n}$. Thus the set of $k \times n$ matrices with complex entries parameterizes $\operatorname{Gr}(k, n)$ via the map

$$
\begin{aligned}
\operatorname{Mat}_{k \times n} & \longrightarrow \operatorname{Gr}(k, n), \\
M & \longmapsto \operatorname{row} \operatorname{space}(M) .
\end{aligned}
$$

This restricts to an injective map from $\operatorname{Mat}_{k \times(n-k)}$ to a dense open set of $\operatorname{Gr}(k, n)$,

$$
\begin{aligned}
\operatorname{Mat}_{k \times(n-k)} & \longrightarrow \operatorname{Gr}(k, n), \\
M & \longmapsto \text { row space }\left(\operatorname{Id}_{k \times k}: M\right),
\end{aligned}
$$

giving local coordinates for the Grassmannian.

Schubert varieties also have local coordinates. The flag $F_{\bullet}(\infty)$ has $i$ dimensional subspace

$$
F_{i}=\operatorname{span}\left\{e_{n+1-i}, \ldots, e_{n-1}, e_{n}\right\},
$$

where $\left\{e_{1}, \ldots, e_{n}\right\}$ are the standard basis vectors of $\mathbb{C}^{n}$. The Schubert variety $X_{\lambda}(\infty)$ has local coordinates given given by matrices $M$ whose entries satisfy

$$
M_{i, j}= \begin{cases}1 & \text { if } j=i+\lambda_{k+1-i}, \\ 0 & \text { if } j=a+\lambda_{k+1-a} \text { for } a \neq i \\ 0 & \text { if } j<i+\lambda_{k+1-i}\end{cases}
$$

and whose other entries are arbitrary complex numbers. For example, $X_{\square}(\infty) \subset \operatorname{Gr}(3,6)$ is parameterized by matrices of the form

$$
\left(\begin{array}{cccccc}
1 & M_{1,2} & 0 & M_{1,4} & 0 & M_{1,6} \\
0 & \square & 1 & M_{2,4} & 0 & M_{2,6} \\
0 & \square & 0 & \square & 1 & M_{3,6}
\end{array}\right) .
$$

Here, $\square$ denotes an entry which is zero.

The flag $F_{\bullet}(0)$ has $F_{i}=\operatorname{span}\left\{e_{1}, \ldots, e_{i}\right\}$ and local coordinates for $X_{\lambda}(0)$ are given by reversing the columns of those for $X_{\lambda}(\infty)$. More interesting 
is that $X_{\lambda}(\infty) \cap X_{\mu}(0)$ has local coordinates given by matrices $M$ whose entries satisfy

$$
M_{i, j}= \begin{cases}1 & \text { if } j=i+\lambda_{k+1-i} \\ 0 & \text { if } j<i+\lambda_{k+1-i}, \\ 0 & \text { if } n+1-i-\mu_{i}<j\end{cases}
$$

and whose other entries are arbitrary complex numbers. For example, $X_{\boxminus}(\infty) \cap X_{\boxminus}(0) \subset \operatorname{Gr}(3,6)$ has local coordinates given by matrices of the form

$$
\left(\begin{array}{cccccc}
1 & M_{1,2} & \square & 0 & \square & 0 \\
0 & \square & 1 & M_{2,4} & \square & 0 \\
0 & \square & 0 & 1 & M_{3,5} & M_{3,6}
\end{array}\right) .
$$

Let us represent a flag $F_{\bullet}$ by a matrix with rows $f_{1}, \ldots, f_{n}$ so that

$$
F_{i}=\operatorname{row} \text { space }\left(\begin{array}{c}
f_{1} \\
\vdots \\
f_{i}
\end{array}\right),
$$

(and also write $F_{i}$ for this matrix). Then the condition (1.1) that $H \in X_{\nu} F_{\bullet}$ is expressed in any of these local coordinates $M$ for $\operatorname{Gr}(k, n), X_{\lambda}(\infty)$, or $X_{\lambda}(\infty) \cap X_{\mu}(0)$ by

$$
\operatorname{rank}\left(\begin{array}{c}
M \\
F_{n-k+i-\nu_{i}}
\end{array}\right) \leq n-\nu_{i} \text { for } i=1, \ldots, k .
$$

Each rank condition is given by the vanishing of all minors of the matrix of size $n-\nu_{i}+1$, and therefore by a system of polynomials in the entries of $M$.

Given an osculating instance of a Schubert problem $\boldsymbol{\lambda}$,

$$
X_{\lambda^{1}}\left(t_{1}\right) \cap X_{\lambda^{2}}\left(t_{2}\right) \cap \cdots \cap X_{\lambda^{m}}\left(t_{m}\right),
$$

the rank equations (2.2) formulate it as a system of polynomials in local coordinates for the Grassmannian. If, say $t_{m}=\infty$, then we may formulate this instance in the smaller set of local coordinates for $X_{\lambda^{m}}(\infty)$, and if we also have $t_{m-1}=0$, a further reduction is possible using the coordinates for $X_{\lambda^{m-1}}(0) \cap X_{\lambda^{m}}(\infty)$.

We entertain these possibilities because solving Schubert problems using symbolic computation is sensitive to the number of variables. Whenever possible, computations in the experiment assume that $\infty$ and 0 are osculation points. If two points of osculation are real, this is achieved by a simple change of variables.

A further sleight of hand is necessary for this to be computationally feasible. Symbolic computation works best over the field $\mathbb{Q}$, and not as well over $\mathbb{Q}[\sqrt{-1}]$. We choose our nonreal osculation points to lie in $\mathbb{Q}[\sqrt{-1}]$, 
but exploit that they come in pairs to formulate equations over $\mathbb{Q}$. Indeed, if $t \notin \mathbb{R}$, then the polynomials $I$ for $X_{\lambda}(t)$ described above will have complex coefficients. Taking real and imaginary parts of the polynomials in $I$ will give real polynomials that define $X_{\lambda}(t) \cap X_{\lambda}(\bar{t})$.

2.3. Methods. This experiment formulates real osculating instances of Schubert problems, determines their number of real solutions, records the result by osculation type, and repeats this hundreds of millions of times on a supercomputer. The overall framework and basic code was adapted from that developed for other experimental projects our group has run to study generalizations of the Shapiro Conjecture $[11,14]$. This experimental design and core code are due to Hillar and are explained in detail in [18].

The experiment was organized around a MySQL database hosted at Texas A\&M University. The database keeps track of all aspects of the experiment, from the problems to be computed (and how they are computed) to the current state of the computation to the data from the computation. We wrote web-based tools to communicate with the database and display the data from the experiment, allowing us to monitor the computations. The computation was controlled by a perl script that, when run, gets a problem to work on from the database, sets up and runs the computation, and upon conclusion, updates the database with its results. The perl script may be run on any machine with access to the database, and we used jobscheduling tools to control its running on the two clusters we have access to at Texas A\&M. These are the brazos cluster in which our research group controls 20 eight-core nodes, and the Calclabs, which consists of over 200 Linux workstations that moonlight as a Beowulf cluster - their day job being calculus instruction. In all, the experiment solved over 344 million real osculating instances of 756 Schubert problems and used 549 gigahertz-years of computing.

A separate program was used to load problems into the database, which were first screened for possible interest and feasibility. During loading, it was also determined which scheme of local coordinates to use for computing that particular problem, similar to the protocol followed in [11, 14]. The actual computation also followed those experiments, and more detail, including references, is given in loc. cit. Briefly, an instance was formulated as a system of polynomials in local coordinates; then Gröbner basis methods implemented in Singular [3] computed a univariate eliminant whose number of real roots is equal to the number of real solutions. These were counted using the symbolic method of Sturm (implemented in Singular's rootsur [30] library).

The experiment was designed to be robust. All calculations are repeatable as the data are deterministically generated from random seeds which are stored in the database. The inevitability of problems, from processor 
failures to power outages to erroneous human intervention with the database, motivated us to build in recoverability from all such events.

2.4. Some results. The results from computing each Schubert problem are recorded in frequency tables such as Table 2 and may be browsed online at [15]. The tables display the number of instances of a given osculation type with a given number of real solutions. This clarifies the dependence of the number of real solutions upon osculation type. For many of the Schubert problems we studied, there is clearly some structure in the possible number of real solutions in terms of osculation type, but this behavior is not uniform across all Schubert problems.

For example, in a few problems there appeared to be an upper bound on the number of real solutions that depends on osculation type, such as the problem $\Xi^{2} \cdot \square^{3}=6$ in $\operatorname{Gr}(3,6)$ of Table 3 . This used 1.4 gigahertz-days

TABLE 3. Frequency table for $\Xi^{2} \cdot \square^{3}=6$ in $\operatorname{Gr}(3,6)$

\begin{tabular}{|l|l||r|r|r|r||r|}
\hline \multirow{2}{*}{$r_{\boxplus}$} & \multirow{2}{*}{$r_{\square}$} & \multicolumn{4}{|c|}{ Number of Real Solutions } & \multirow{2}{*}{ Total } \\
\cline { 3 - 6 } & & 0 & 2 & 4 & 6 & \\
\hline \hline 2 & 3 & & & & 100000 & 100000 \\
\hline 2 & 1 & 27855 & 11739 & 22935 & 37471 & 100000 \\
\hline 0 & 3 & 17424 & 82576 & & & 100000 \\
\hline 0 & 1 & & 100000 & & & 100000 \\
\hline
\end{tabular}

of computing. For the vast majority of the Schubert problems there were instances of every osculation type with all solutions real, and it was not clear what distinguished this second class of Schubert problems from the first. In Section 4 we present tables from several other Schubert problems and discuss other structures we observe.

Of the 756 Schubert problems studied, 273 had the form (1.8) and so had a topological lower bound given by Proposition 4 . These included the Wronski maps for $\operatorname{Gr}(2,4), \operatorname{Gr}(2,6)$, and $\operatorname{Gr}(2,8)$ for which Eremenko and Gabrielov had shown that the lower bound of zero was sharp [7]. For 264 of the remaining 270 cases, instances were computed showing that this topological lower bound was sharp. There were however six Schubert problems for which the topological lower bounds were not observed. These were

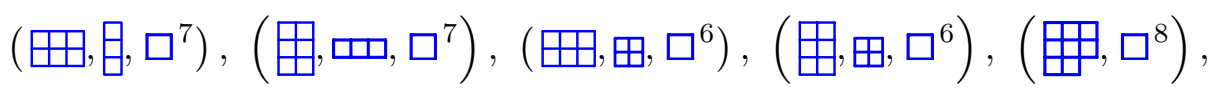

all in $\operatorname{Gr}(4,8)$, and $\square^{9}$ in $\operatorname{Gr}(3,6)$. These have observed lower bounds of $3,3,2,2,2,2$ and sign-imbalances of $1,1,0,0,0,0$, respectively. There is not yet an explanation for the first four, but the last two are symmetric 
Schubert problems, which were observed to have a congruence modulo four on their numbers of real solutions. This congruence gives a lower bound of two for both problems $\mathbb{}$. $\square^{8}=90$ in $\operatorname{Gr}(4,8)$ and $\square^{9}=42$ in $\operatorname{Gr}(3,6)$.

Table 4 shows the result of computing a million real osculating instances of the Schubert problem $\square^{9}=42$ in $\operatorname{Gr}(3,6)$, which used 1.07 gigahertzyears of computing. As with Table 1, only numbers of real solutions con-

TABLE 4. Frequency table for $\square^{9}=42$ in $\operatorname{Gr}(3,6)$

\begin{tabular}{|c|c|c|c|c|c|c|c|c|c|c|c|c|}
\hline \multirow{2}{*}{$r_{\square}$} & \multicolumn{12}{|c|}{ Number of Real Solutions } \\
\hline & 0 & 2 & 4 & 6 & 8 & 10 & 12 & 14 & 16 & 18 & 20 & $\cdots$ \\
\hline 9 & & & & & & & & & & & & $\ldots$ \\
\hline 7 & & 1843 & & 13286 & & 69319 & & 18045 & & 13998 & & $\cdots$ \\
\hline 5 & & 30223 & & 51802 & & 57040 & & 17100 & & 12063 & & $\cdots$ \\
\hline 3 & & 34314 & & 93732 & & 47142 & & 10213 & & 5532 & & $\cdots$ \\
\hline 1 & & & & 151847 & & 35220 & & 6416 & & 2931 & & $\cdots$ \\
\hline
\end{tabular}

\begin{tabular}{|l|r|r|r|r|r|r|r|r|r|r|r||r|}
\hline \multicolumn{1}{c|}{ Number of Real Solutions } & \multirow{2}{*}{ Total } \\
\hline$\cdots$ & 22 & 24 & 26 & 28 & 30 & 32 & 34 & 36 & 38 & 40 & 42 & \\
\hline$\cdots$ & & & & & & & & & & & 200000 & 200000 \\
\hline$\cdots$ & 22883 & & 4592 & & 11603 & & 3891 & & 473 & & 40067 & 200000 \\
\hline$\cdots$ & 15220 & & 2767 & & 4634 & & 2056 & & 211 & & 6884 & 200000 \\
\hline$\cdots$ & 5492 & & 839 & & 1194 & & 504 & & 65 & & 973 & 200000 \\
\hline$\cdots$ & 2345 & & 362 & & 450 & & 181 & & 22 & & 226 & 200000 \\
\hline
\end{tabular}

gruent to 42 modulo four were observed.

The observed congruence modulo four which were inspired by these computations (and those of the earlier investigation [13]) were established in $[16,17]$.

A partition $\lambda$ is symmetric if it equals its matrix-transpose. For example, all except the last of the following are symmetric,

$$
\text { 口, 巴, 田, 巴, 曲, 曲, 目, 册, 巴。 }
$$

A Schubert problem $\boldsymbol{\lambda}$ in $\operatorname{Gr}(k, 2 k)$ is symmetric if every partition in $\boldsymbol{\lambda}$ is symmetric. For a symmetric partition $\lambda$, let $\ell(\lambda)$ be the number of boxes in its main diagonal, which is the maximum number $i$ with $\lambda_{i} \geq i$. We state the main result of $[16,17]$.

Proposition 5. Suppose that $\boldsymbol{\lambda}=\left(\lambda^{1}, \ldots, \lambda^{m}\right)$ is a symmetric Schubert problem in $\operatorname{Gr}(k, 2 k)$ with $\sum_{i} \ell\left(\lambda^{i}\right) \geq k+4$. Then the number of real solutions to a real osculating instance of $\boldsymbol{\lambda}$ is congruent to the number of complex solutions modulo four. 
One of the symmetric problems, 囲 $\cdot \square^{7}=20$ in $\operatorname{Gr}(4,8)$, not only exhibited this congruence but also appeared to have lower bounds depending upon the osculation type $r_{\square}$ as well as further gaps in its numbers of real solutions (we never observed 12 or 16 real solutions). Table 5 displays the result of computing 400000 real osculating instances of this Schubert prob-

TABLE 5. Gaps and lower bounds for 囲 $\square^{7}=20$ in $\operatorname{Gr}(4,8)$

\begin{tabular}{|r||r|r|r|r|r|r|r|r|r|r|r||r|}
\hline \multicolumn{1}{|c||}{$r_{\square}$} & \multicolumn{10}{|c|}{ Total } \\
\cline { 2 - 11 } & 0 & 2 & 4 & 6 & 8 & 10 & 12 & 14 & 16 & 18 & 20 & \\
\hline \hline 7 & & & & & & & & & & & 100000 & 100000 \\
\hline 5 & & & & & 85080 & & & & & & 14920 & 100000 \\
\hline 3 & & & 66825 & & 30232 & & & & & & 2943 & 100000 \\
\hline 1 & 37074 & & 47271 & & 14517 & & & & & & 1138 & 100000 \\
\hline
\end{tabular}

lem, which used 2.06 gigahertz-days of computing. This is a member of a family of Schubert problems (the problem of Table 2 is another) that we can solve completely, and whose numbers of real solutions have a lower bound depending on osculation type, as well as gaps. We explain this in the next section.

\section{Lower bounds via factorization}

In our experimentation, we saw that Schubert problems related to the two problems $\square \square \square \cdot \square^{7}=6$ in $\operatorname{Gr}(2,8)$ and 团 $\cdot \square^{7}=20$ in $\operatorname{Gr}(4,8)$ (from Tables 2 and 5, respectively) appeared to have gaps and lower bounds depending on $r_{\square}$ in their numbers of real solutions. These are members of a family of Schubert problems, one for each Grassmannian $\operatorname{Gr}(k, n)$ with $2 \leq k, n-k$, which we are able to solve completely, thereby determining all possibilities for the number of real solutions and explaining these gaps and lower bounds.

For $k, n$, let $\boxplus_{k, n}$ ( $\boxplus$ for short) denote the partition $\left((n-k-1)^{k-1}, 0\right)$ ( $n-k-1$ repeated $k-1$ times), which is the complement of a full hook, $\left(n-k, 1^{k-1}\right)$. For example,

$$
\boxplus_{2,6}=\square, \quad \boxplus_{3,8}=\boxplus \text { 口, and } \boxplus_{4,8}=\text { 田. }
$$

The osculating Schubert problems in this family all have the form $\boldsymbol{\lambda}=$ $\left(\boxplus, \square^{n-1}\right)$ in $\operatorname{Gr}(k, n)$, and they all have topological lower bounds $\sigma\left(\mathbb{\boxplus}_{k, n}\right)$ coming from Proposition 4. The multinomial coefficient $\left(\begin{array}{c}n \\ a, b\end{array}\right)$ is zero unless $n=a+b$, and in that case it equals $\frac{n !}{a ! b !}$. 
Lemma 6. The Schubert problem $\boldsymbol{\lambda}=\left(\boxplus, \square^{n-1}\right)$ in $\operatorname{Gr}(k, n)$ has $\left(\begin{array}{l}n-2 \\ k-1\end{array}\right)$ solutions and $\sigma(\boxplus)=\left(\begin{array}{c}\left\lfloor\frac{n-2}{2}\right\rfloor \\ \left\lfloor\frac{k-1}{2}\right\rfloor,\left\lfloor\frac{n-k-1}{2}\right\rfloor\end{array}\right)$, which is zero unless $n$ is even and $k$ is odd.

Proof. The number of solutions of the Schubert problem $\boldsymbol{\lambda}=\left(\mathbb{\boxplus}, \square^{n-1}\right)$ in $\operatorname{Gr}(k, n)$ is the number of Young tableaux of shape $\boxplus^{c}$, which is a full hook $\left(n-k, 1^{k-1}\right)$ consisting of one row of length $n-k$ and one column of length $k$. Here are full hooks for $(k, n)$ equal to $(2,6),(3,8),(4,8)$, and $(4,10)$.

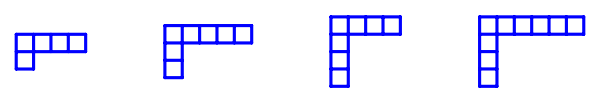

A tableau with such a hook shape has a 1 in its upper left box and the numbers $2, \ldots, n-1$ filling out its first row and first column. This filling is determined by the $k-1$ numbers in the rest of its first column. Thus there are $\left(\begin{array}{l}n-2 \\ k-1\end{array}\right)$ tableaux of hook shape $\left(n-k, 1^{k-1}\right)$.

Reading a tableau $T$ with hook shape gives a word of the form $1 A B$, where $1 A$ is the first row and $1 B$ is the first column. This is the permutation corresponding to $T$ whose sign contributes to the sign-imbalance. The subwords $A B$ are shuffles of the numbers $\{2, \ldots, n-1\}$. Counting these permutations by their lengths is the evaluation of the Gaussian polynomial $\left(\begin{array}{l}n-2 \\ k-1\end{array}\right)_{q}$ at $q=-1$. Thus the sign imbalance is $\left(\begin{array}{l}n-2 \\ k-1\end{array}\right)_{-1}$, which is well-known (see e.g. [25, Prop. 7.10]) to be $\left(\begin{array}{c}\left\lfloor\frac{n-2}{2}\right\rfloor \\ \left\lfloor\frac{k-1}{2}\right\rfloor,\left\lfloor\frac{n-k-1}{2}\right\rfloor\end{array}\right)$.

Theorem 7. For any $k, n$, the solutions to the osculating instance of the Schubert problem ( $\left.\boxplus, \square^{n-1}\right)$ in $\operatorname{Gr}(k, n)$,

$$
X_{\square}\left(t_{1}\right) \cap X_{\square}\left(t_{2}\right) \cap \cdots \cap X_{\square}\left(t_{n-1}\right) \cap X_{\text {巴 }}(\infty),
$$

may be identified with all ways of factoring $f^{\prime}(t)=g(t) h(t)$ where

$$
f(t)=\prod_{i=1}^{n-1}\left(t-t_{i}\right)
$$

with $\operatorname{deg} g=k-1$ and $\operatorname{deg} h=n-k-1$ are monic.

By this theorem, the number of real solutions to a real osculating instance of the Schubert problem ( $\mathbb{\Psi}, \square^{n-1}$ ) with osculation type $r_{\square}$ will be the number of real factorizations $f^{\prime}(t)=g(t) h(t)$ where $f(t)$ has exactly $r_{\square}$ real roots, $\operatorname{deg} g=n-k-1$, and $\operatorname{deg} h=k-1$. This counting problem was studied in [25, Sect. 7], and we recount it here. Let $r$ be the number of real roots of $f^{\prime}(t)$. By Rolle's Theorem, $r_{\square}-1 \leq r \leq n-2$. Then the number $\nu(k, n, r)$ of such factorizations is the coefficient of $x^{n-k-1} y^{k-1}$ in 
$(x+y)^{r}\left(x^{2}+y^{2}\right)^{c}$, where $c=\frac{n-2-r}{2}$ is the number of irreducible quadratic factors of $f^{\prime}(t)$.

Corollary 8. The number of real solutions to a real osculating instance of the Schubert problem $\left(\boxplus, \square^{n-1}\right)$ (3.1) with osculation type $r_{\square}$ is $\nu(k, n, r)$, with $r$ the number of real roots of $f^{\prime}(t)$, where $f$ is the polynomial (3.2).

Remark 9. When $r<n-2$, we have that $\nu(k, n, r) \leq \nu(k, n, r+2)$, so $\nu\left(k, n, r_{\square}-1\right)$ is the lower bound for the number of real solutions to a real osculating instance of $\left(\boxplus, \square^{n-1}\right)$ of osculation type $r_{\square}$. Since at most $\left\lfloor\frac{n}{2}\right\rfloor$ different values of $r$ may occur for the numbers of real roots of $f^{\prime}(t)$, the number $\nu(k, n, r)$ satisfies

$$
\left(\begin{array}{c}
\left\lfloor\frac{n-2}{2}\right\rfloor \\
\left\lfloor\frac{k-1}{2}\right\rfloor,\left\lfloor\frac{n-k-1}{2}\right\rfloor
\end{array}\right) \leq \nu(k, n, r) \leq\left(\begin{array}{l}
n-2 \\
k-1
\end{array}\right) .
$$

There will in general be lacunae in the numbers of real solutions, as we saw in Table 5. For example, the values of $\nu(5,13, r)$ are

$$
10,18,38,78,162 \text {, and } 330 \text {. }
$$

Remark 10. The Schubert problem ( $\left.⿴ 囗 十, \square^{2 k-1}\right)$ in $\operatorname{Gr}(k, 2 k)$ is symmetric. When $k>2$ it satisfies the hypotheses of Proposition 5 and so its numbers of real solutions (the numbers $\nu(k, 2 k, r))$ are congruent to $\left(\begin{array}{c}2 k-2 \\ k-1\end{array}\right)$ modulo four. We deduce this congruence modulo four from Theorem 7 by proving that the number of nonreal solutions to a real osculating instance of such a Schubert problem is a multiple of four. Equivalently, given a real polynomial $\phi(t)$ of degree $2 m=2 k-2$ with distinct roots, the number of nonreal ordered pairs $(g(t), h(t))$ of polynomials of degree $m$ with $\phi(t)=g(t) h(t)$ is a multiple of four.

An ordered pair $(g(t), h(t))$ of polynomials of degree $m$ with $\phi(t)=$ $g(t) h(t)$ is an ordered factorization of $\phi(t)$. Given a factorization $\phi(t)=$ $g(t) h(t)$, we have $\phi(t)=h(t) g(t)$, and so $(g(t), h(t))$ and $(h(t), g(t))$ are distinct ordered factorizations of $\phi(t)$. If $g(t)$ (and hence $h(t))$ is not real, and we do not have $\overline{g(t)}=h(t)$, then

$$
(g(t), h(t)),(h(t), g(t)),(\overline{g(t)}, \overline{h(t)}),(\overline{h(t)}, \overline{g(t)})
$$

are four distinct nonreal ordered factorizations.

To show that the set of nonreal ordered factorizations of $\phi(t)$ is divisible by four, we need only to show that the number for which $\overline{g(t)}=h(t)$ is a multiple of four. These can only occur when $\varphi(t)$ has no real roots, for $g(t)$ must have one root from each complex conjugate pair of roots of $\varphi(t)$. There are $2^{m}$ such pairs and so $2^{m}$ such factorizations, which is a multiple of four when $m>1$ and thus when $k>2$. This establishes the congruence 
modulo four of Proposition 5 for osculating instances of Schubert problems $\left(\boxplus, \square^{2 k-1}\right)$ in $\operatorname{Gr}(k, 2 k)$ when $k>2$.

It is interesting to note that by (3.3), the number of real solutions to this problem in $\operatorname{Gr}(5,13)$ also satisfies a congruence modulo four.

Proof of Theorem 7 . The Schubert variety $X_{\text {王 }}(\infty)$ consists of those $H$ satisfying

$$
\operatorname{dim} H \cap F_{i+1}(\infty) \geq i \quad \text { for } i=1, \ldots, k-1 .
$$

By Proposition 1, the solutions to (3.1) will be points in $X_{\boxplus}(\infty)$ that do not lie in any other smaller Schubert variety $X_{\lambda}(\infty)$. This is the Schubert cell of $X_{\boxplus}(\infty)$ [10], and it consists of the $k$-planes $H$ which are row spaces of matrices of the form

$$
\left(\begin{array}{cccccccc}
1 & x_{1} & \cdots & x_{n-k-1} & x_{n-k} & 0 & \cdots & 0 \\
0 & 0 & \cdots & 0 & 1 & x_{n-k+1} & \cdots & 0 \\
\vdots & \vdots & & \vdots & & \ddots & \ddots & \vdots \\
0 & 0 & \cdots & 0 & \cdots & 0 & 1 & x_{n-1}
\end{array}\right),
$$

where $x_{1}, \ldots, x_{n-1}$ are indeterminates. If $x_{n-k}=0$, then $H \in X_{\square}(0)$, but if one of $x_{n-k+1}, \ldots, x_{n-1}$ vanishes, then $H \in X_{\theta}(0)$, which cannot occur for a solution to (3.1), again by Proposition 1.

We use a rescaling of these coordinates. Define constants $g_{n-k-1}:=$ $1=: h_{k-1}$ and $c_{i}:=(-1)^{n-k-i+1}(n-k-i) !$ and let $(f, g, h)=\left(f_{0}, g_{0}, \ldots\right.$, $\left.g_{n-k-1}, h_{0}, \ldots, h_{k-2}\right)$ be variables with $h_{0}, \ldots, h_{k-2}$ all nonzero. If we let $H(f, g, h)$ be the row space of the following matrix (also written $H(f, g, h)$ ):

$$
\left(\begin{array}{ccccccccc}
c_{1} g_{n-k-1} & c_{2} g_{n-k-2} & \cdots & c_{n-k} g_{0} & \frac{f_{0}}{h_{0}} & 0 & \cdots & 0 & 0 \\
0 & 0 & \cdots & 0 & -1 & \frac{h_{0}}{h_{1}} & \cdots & 0 & 0 \\
0 & 0 & \cdots & 0 & 0 & -2 & \ddots & \vdots & \vdots \\
\vdots & \vdots & & \vdots & \vdots & & \ddots & & 0 \\
0 & 0 & \cdots & 0 & 0 & \cdots & -(k-2) & \frac{h_{k-3}}{h_{k-2}} & 0 \\
0 & 0 & \cdots & 0 & 0 & \cdots & 0 & -(k-1) & \frac{h_{k-2}}{h_{k-1}}
\end{array}\right)
$$

then $H(f, g, h)$ parameterizes the Schubert cell of $X_{\text {甲 }}(\infty)$. We postpone the following calculation.

Lemma 11. The condition for $H(f, g, h)$ to lie in $X_{\square}(t)$ is

$$
\operatorname{det}\left(\begin{array}{c}
H(f, g, h) \\
F_{n-k}(t)
\end{array}\right)=(-1)^{k(n-k)}\left(\sum_{i=0}^{n-k-1} \sum_{j=0}^{k-1} \frac{t^{i+j+1}}{i+j+1} g_{i} h_{j}+f_{0}\right) \text {. }
$$


Call the polynomial in the parentheses $f(t)$. If $H$ lies in the intersection (3.1), then $(-1)^{k(n-k)} f$ is the polynomial (3.2). If we set

$$
\begin{aligned}
& g(t):=g_{0}+t g_{1}+\cdots+t^{n-k-1} g_{n-k-1} \quad \text { and } \\
& h(t):=h_{0}+t h_{1}+\cdots+t^{k-1} h_{k-1},
\end{aligned}
$$

then we have $f(0)=f_{0}$ and $f^{\prime}(t)=g(t) h(t)$. Theorem 7 is now immediate.

Proof of Lemma 11. Expand the determinant (3.5) along its first $k$ rows (the rows of $H(f, g, h))$ to obtain

$$
\operatorname{det}\left(\begin{array}{c}
H(f, g, h) \\
F_{n-k}(t)
\end{array}\right)=\sum_{\alpha \in\left(\begin{array}{c}
{[n]} \\
k
\end{array}\right)}(-1)^{|\alpha|} H(f, g, h)_{\alpha}\left(F_{n-k}(t)\right)_{\alpha^{c}}
$$

where $\left(\begin{array}{c}{[n]} \\ k\end{array}\right)$ is the collection of subsets of $\{1, \ldots, n\}$ of cardinality $k,|\alpha|:=$ $\alpha_{1}+\cdots+\alpha_{k}-1-\cdots-k, H_{\alpha}$ is the determinant of the $k \times k$ submatrix of $H$ given by the columns in $\alpha$, and $\left(F_{n-k}(t)\right)_{\alpha^{c}}$ is the determinant of the $(n-k) \times(n-k)$ submatrix of $F_{n-k}(t)$ formed by the columns in $\alpha^{c}:=$ $\{1, \ldots, n\} \backslash \alpha$. These are minors of $H(f, g, h)$ and $F_{n-k}(t)$.

A minor $H(f, g, h)_{\alpha}$ of $H(f, g, h)$ is nonzero only if $\alpha=(i, n-k+1, \ldots$, $\widehat{n-k+j}, \ldots, n)$ for $i \in\{1, \ldots, n-k\}$ and $j \in\{1, \ldots, k\}$ or $\alpha=(n-k+1, \ldots$, $n-1, n)$, the last $k$ columns. Write $[i, \widehat{j}]$ for the first type and $[n-k]^{c}$ for the second. Then $\left([n-k]^{c}\right)^{c}=(1, \ldots, n-k)$ and $[\widehat{i}, j]:=[i, \widehat{j}]^{c}=$ $(1, \ldots, \widehat{i}, \ldots, n-k, n-k+j)$.

With $g_{n-k-1}=1=h_{k-1}$, a calculation shows that

$$
\begin{aligned}
H_{[n-k]^{c}} & =f_{0} \quad \text { and } \\
H_{[i, \widehat{j}]} & =(-1)^{n-k-i+j}(n-k-i) !(j-1) ! g_{n-k-i} h_{j-1} .
\end{aligned}
$$

For any $\alpha \in\left(\begin{array}{c}{[n]} \\ n-k\end{array}\right)$ the minor $\left(F_{n-k}(t)\right)_{\alpha}$ is

$$
\operatorname{det}\left(\begin{array}{ccc}
\frac{t^{\alpha_{1}-1}}{\left(\alpha_{1}-1\right) !} & \cdots & \frac{t^{\alpha_{n-k}-1}}{\left(\alpha_{n-k}-1\right) !} \\
\vdots & \ddots & \vdots \\
\frac{t^{\alpha_{1}-(n-k)}}{\left(\alpha_{1}-(n-k)\right) !} & \cdots & \frac{t^{\alpha_{n-k}-(n-k)}}{\left(\alpha_{n-k}-(n-k)\right) !}
\end{array}\right) .
$$


We use (1.5) to compute this determinant. It is

$$
\begin{gathered}
\frac{t^{|\alpha|}}{\left(\alpha_{1}-1\right) ! \cdots\left(\alpha_{n-k}-1\right) !} \operatorname{det}\left(\begin{array}{ccc}
1 & \cdots & 1 \\
\alpha_{1}-1 & \cdots & \alpha_{n-k}-1 \\
\vdots & \ddots & \vdots \\
\left(\alpha_{1}-1\right)_{n-k-1} & \cdots & \left(\alpha_{n-k}-1\right)_{n-k-1}
\end{array}\right) \\
=\frac{t^{|\alpha|}}{\left(\alpha_{1}-1\right) ! \cdots\left(\alpha_{n-k}-1\right) !} \operatorname{det}\left(\begin{array}{ccc}
\alpha_{1} & \cdots & \alpha_{n-k} \\
\vdots & \ddots & \vdots \\
\alpha_{1}^{n-k-1} & \cdots & \alpha_{n-k}^{n-k-1}
\end{array}\right)
\end{gathered}
$$

where $(m)_{i}:=m(m-1) \cdots(m-i+1)$ and an entry in the first matrix is zero if $\alpha_{i}-j<0$. If $\alpha$ ! :=( $\left.\alpha_{1}-1\right) ! \cdots\left(\alpha_{n-k}-1\right)$ !, then

$$
\left(F_{n-k}(t)\right)_{\alpha}=\frac{t^{|\alpha|}}{\alpha !} \prod_{i<j}\left(\alpha_{j}-\alpha_{i}\right)=\frac{t^{|\alpha|}}{\alpha !} \operatorname{Vdm}(\alpha),
$$

where $\operatorname{Vdm}(\alpha)$ is the Vandermonde determinant of $\alpha$. We compute

$$
\begin{aligned}
|[i, \widehat{j}]| & =i+n-k+1+\cdots+n-(n-k+j)-1-\cdots-k \\
& =k(n-k)-(n-k-i+j) \\
\mid \widehat{i}, j] \mid & =1+\cdots+n-k+n-k+j-1-\cdots-k=n-k-i+j \\
([\widehat{i}, j]) ! & =1 ! \cdot 2 ! \cdots(i-2) ! i !(i+1) ! \cdots(n-k-1) !(n-k+j-1) ! \\
\operatorname{Vdm}([\widehat{i}, j]) & =1 ! \cdot 2 ! \cdots(i-2) ! \frac{i !(i+1) !}{1} \cdots \frac{(n-k-1) !}{n-k-i} \\
& =([\widehat{i}, j]) ! \cdot \frac{(n-k+j-1) !}{(n-k-i) !(j-1) !(n-k-i+j)} \cdot \frac{1}{n-k-i+j},
\end{aligned}
$$

and

$$
\left|[n-k]^{c}\right|=k(n-k), \quad[n-k] !=\operatorname{Vdm}([n-k])=1 ! \cdot 2 ! \cdots(n-k-1) ! .
$$

After some cancellation, the determinant (3.6) becomes

$$
\begin{aligned}
\sum_{i=1}^{n-k} \sum_{j=1}^{k}(-1)^{|[i, \hat{j}]|} H(f, g, h)_{[i, \widehat{j}]}\left(F_{n-k}(t)\right)_{\widehat{[i}, j]} \\
+(-1)^{\left|[n-k]^{c}\right|} H(f, g, h)_{[n-k]^{c}}\left(F_{n-k}(t)\right)_{[n-k]} \\
+
\end{aligned}
$$


which is

$$
\sum_{i=1}^{n-k} \sum_{j=1}^{k}(-1)^{k(n-k)} \frac{t^{n-k-i+j}}{n-k-i+j} g_{n-k-i} h_{j-1}+(-1)^{k(n-k)} f_{0} .
$$

If we replace $n-k-i$ by $i$ and $j-1$ by $j$ in this sum, we get

$$
\operatorname{det}\left(\begin{array}{c}
H(f, g, h) \\
F_{n-k}(t)
\end{array}\right)=(-1)^{k(n-k)}\left(\sum_{i=0}^{n-k-1} \sum_{j=0}^{k-1} \frac{t^{i+j+1}}{i+j+1} g_{i} h_{j}+f_{0}\right),
$$

which completes the proof.

\section{More tables from the experiment}

We present a selection of the tables of real osculating instances of Schubert problems studied in [13] and [15]. These exhibit intriguing structures in their numbers of real solutions, only some of which we understand.

4.1. An enigma. Table 6 shows what is perhaps the most complicated structure we observed. This used 24.6 gigahertz-years of computing. The

\begin{tabular}{|c|c|c|c|c|c|c|c|c|c|c|c|c|c|}
\hline \multirow{2}{*}{${ }^{r} \boxplus$} & \multirow[b]{2}{*}{$r_{\square}$} & \multicolumn{12}{|c|}{ Number of Real Solutions } \\
\hline & & \multirow[t]{2}{*}{0} & 2 & \multirow{2}{*}{\multicolumn{2}{|c|}{4}} & \multicolumn{2}{|c|}{6} & \multicolumn{2}{|c|}{8} & 10 & 12 & 14 & \multirow{2}{*}{\begin{tabular}{|l|}
$\cdots$ \\
$\ldots$
\end{tabular}} \\
\hline 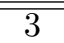 & 2 & & \multirow[b]{2}{*}{2714} & & \multirow{2}{*}{\multicolumn{3}{|c|}{111636}} & & & & & & \\
\hline 3 & 0 & 5 & & 1304 & & & & \multicolumn{2}{|c|}{59800} & 88674 & 20255 & 52088 & \multirow{2}{*}{\begin{tabular}{|l|} 
\\
\end{tabular}} \\
\hline 1 & 2 & 81216 & 235048 & 7268 & \multirow{2}{*}{\multicolumn{3}{|c|}{109908}} & 960 & & & 2877 & 12685 & \\
\hline 1 & 0 & & 599421 & & \multicolumn{2}{|c|}{83350} & & & 53394 & & & 20997 & $\cdots$ \\
\hline \multicolumn{14}{|c|}{ Number of Real Solutions } \\
\hline$\cdots$ & 16 & 18 & 20 & 22 & \multicolumn{2}{|c|}{\begin{tabular}{|l|l}
24 \\
\end{tabular}} & & 26 & 28 & 8 & 32 & 34 & $\cdots$ \\
\hline$\cdots$ & & & & & & & & & & & & & $\bar{\cdots}$ \\
\hline$\cdots$ & 44306 & 164085 & 9467 & 23019 & 52 & 22 & 271 & 149 & 5044 & 1695 & 1107 & 6336 & $\cdots$ \\
\hline$\cdots$ & 4953 & 31084 & 10 & 50198 & & & 1664 & 418 & & & & & $\cdots$ \\
\hline$\cdots$ & & 20896 & & 16359 & & & 345 & 543 & & & & & $\cdots$ \\
\hline & & & $\mathrm{Nu}$ & mber of & f Rea & l Sol & lutic & & & & & & Catol \\
\hline$\cdots$ & 36 & 38 & \begin{tabular}{l|l}
40 & \\
\end{tabular} & 42 & 44 & 46 & \begin{tabular}{l|l}
6 & 4 \\
\end{tabular} & 48 & 50 & 52 & 54 & 4 & otal \\
\hline$\cdots$ & & & & & & & & & & & 828960 & & 8960 \\
\hline$\cdots$ & 1280 & 15495 & 1731 & 13362 & 240 & 6292 & & & 35970 & 1275 & 102406 & 828 & 3960 \\
\hline$\cdots$ & & & & & & & & & & & & 828 & 3960 \\
\hline$\cdots$ & & & & & & & & & & & & 828 & 8960 \\
\hline
\end{tabular}

TABLE 6. Frequency table for $⿴ 囗 十 \cdot \boxplus^{3} \cdot \square^{2}=54$ in $\operatorname{Gr}(4,8)$ 
first row has only real points of osculation, so the Mukhin-Tarasov-Varchenko Theorem implies that all 54 solutions are real, as observed. All possible numbers of real solutions except 48 were observed for the osculation type of the second row. The third and fourth rows appear to have an upper bound of 26 , and the fourth row exhibits an additional congruence to 54 modulo four. None of this, besides the first row, is understood. Compare the upper bound for the last two rows to that in Table 3 (note that $2=2 \cdot\lfloor 6 / 4\rfloor$ and $26=2 \cdot\lfloor 54 / 4\rfloor)$. A similar structure was also observed for the Schubert problem $⿴ 囗 十 \cdot \boxplus \cdot \Xi^{2} \cdot \square=16$ in $\operatorname{Gr}(4,8)$.

4.2. Internal structure. Work of Vakil [31] and others has led to the study of Schubert problems which posses internal structure as encoded by their Galois groups [12]. The current state of this investigation is discussed in $[4, \S 5]$. Intriguingly, in every problem we know whose Galois group is not the full symmetric group, the internal structure which restricts the Galois group appears to restrict the possible numbers of real solutions to real osculating instances.

A good example is the Schubert problem ${ }^{2} \cdot$ 睡 $^{2} \square^{6}=10$ in $\operatorname{Gr}(4,9)$ of Table 7, which used 4.14 gigahertz-years of computing. The problem is solved by first solving an instance of the problem of four lines $\left(\square^{4}=2\right)$ in a $\operatorname{Gr}(2,4)$ that is given by the four conditions ${ }^{2} \cdot \mathrm{I}^{2}$. Then, for each of the

TABLE 7. Frequency table for $\mathbf{m}^{2} \cdot \theta^{2} \cdot \square^{6}=10$ in $\operatorname{Gr}(4,9)$

\begin{tabular}{|c|c|c|c|c|c|c|c|c|c|}
\hline \multirow{2}{*}{$r_{\mathrm{m}}^{\mathrm{m}}$} & \multirow{2}{*}{$r_{\text {目 }}$} & \multirow{2}{*}{$r_{\square}$} & \multicolumn{6}{|c|}{ \# Real Solutions } & \multirow{2}{*}{ Total } \\
\hline & & & 0 & 2 & 4 & 6 & 8 & 10 & \\
\hline 2 & 2 & 6 & & & & & & 8000 & 8000 \\
\hline 2 & 0 & 6 & 5419 & & & & & 2581 & 8000 \\
\hline 0 & 2 & 6 & 2586 & & & & & 5414 & 8000 \\
\hline 0 & 0 & 6 & & & & & & 8000 & 8000 \\
\hline 2 & 2 & 4 & & 2971 & & 2202 & & 2827 & 8000 \\
\hline 2 & 0 & 4 & 5508 & 876 & & 722 & & 894 & 8000 \\
\hline 0 & 2 & 4 & 2469 & 2051 & & 1527 & & 1953 & 8000 \\
\hline 0 & 0 & 4 & & 2941 & & 2228 & & 2831 & 8000 \\
\hline 2 & 2 & 2 & & 3595 & & 3374 & & 1031 & 8000 \\
\hline 2 & 0 & 2 & 5535 & 1090 & & 1051 & & 324 & 8000 \\
\hline 0 & 2 & 2 & 2539 & 2472 & & 2254 & & 735 & 8000 \\
\hline 0 & 0 & 2 & & 3572 & & 3411 & & 1017 & 8000 \\
\hline 2 & 2 & 0 & & & & 7287 & & 713 & 8000 \\
\hline 2 & 0 & 0 & 5378 & & & 2386 & & 236 & 8000 \\
\hline 0 & 2 & 0 & 2619 & & & 4917 & & 464 & 8000 \\
\hline 0 & 0 & 0 & & & & 7333 & & 667 & 8000 \\
\hline
\end{tabular}


two solutions to that problem, an instance of the Schubert problem $\square^{6}=5$ in $\operatorname{Gr}(2,5)$ is solved, to get 10 solutions in all. The Galois group of this problem permutes each of these blocks of five solutions for the two Schubert problems $\square^{6}=5$ of the second step, and thus it acts imprimitively. Further analysis shows that the Galois group is the wreath product $S_{5} 2 S_{2}$, which has order $(5 !)^{2} \cdot 2 !=28800$.

Table 8 shows the frequency tables for the two auxiliary problems $\square^{4}=2$ in $\operatorname{Gr}(2,4)$ and $\square^{6}=5$ in $\operatorname{Gr}(2,5)$, which used 12.6 gigahertz-hours of computing. It is fascinating to compare these to Table 7. First observe

TABLE 8. Frequency tables for $\square^{4}=2$ and $\square^{6}=5$.

\begin{tabular}{|c||r|r||r|}
\hline$r_{\square}$ & 0 & 2 & Total \\
\hline \hline 4 & & 100000 & 100000 \\
\hline 2 & 32412 & 67588 & 100000 \\
\hline 0 & & 100000 & 100000 \\
\hline
\end{tabular}

\begin{tabular}{|c||r|r|r||r|}
\hline$r_{\square}$ & 1 & 3 & 5 & Total \\
\hline \hline 6 & & & 100000 & 100000 \\
\hline 4 & 36970 & 36970 & 35314 & 100000 \\
\hline 2 & 35314 & 43081 & 11222 & 100000 \\
\hline 0 & & 89105 & 10895 & 100000 \\
\hline
\end{tabular}

that for $\mathrm{m}^{2} \cdot \mathrm{B}^{2} \cdot \square^{6}$ we have no real solutions only when $r_{\mathrm{m}}+r_{\text {目 }}=2$, similar to $\square^{4}$ having no real solutions only when $r_{\square}=2$. The remaining columns of Table 7 have the same pattern of dependence on $r_{\square}$ as do the columns of $\square^{6}=5$, except that the number of real solutions is doubled.

4.3. Problems of the form $(\lambda, \lambda, \lambda, \lambda)$. When the Schubert problem has the form $\boldsymbol{\lambda}=\lambda^{4}$, there are three osculation types, $r_{\lambda}=4, r_{\lambda}=2$, and $r_{\lambda}=0$. Every Schubert problem of this type we studied has interesting structure in its numbers of real solutions. Table 9 shows the results for the

TABLE 9. Frequency table for $\boxplus^{4}=8$ in $\operatorname{Gr}(3,7)$.

\begin{tabular}{|c||r|r|r|r|r||r|}
\hline \multicolumn{1}{|c||}{$r_{\boxplus}$} & \multicolumn{5}{c||}{ \# Real Solutions } & \multirow{2}{*}{ Total } \\
\cline { 2 - 6 } & 0 & 2 & 4 & 6 & 8 & \\
\hline \hline 4 & & & & & 10000 & 10000 \\
\hline 2 & 3590 & 292 & 6118 & & & 10000 \\
\hline 0 & & & 10000 & & & 10000 \\
\hline
\end{tabular}

Schubert problem $\Xi^{4}=8$ in $\operatorname{Gr}(3,7)$. The structure of this table is similar to Table 10 for the Schubert problem $\Psi^{4}=9$ in $\operatorname{Gr}(4,8)$. Both of these were computed in [13] which inspired the more comprehensive experiment we have been discussing. 
TABLE 10. Frequency table for $\square^{4}=9$ in $\operatorname{Gr}(4,8)$.

\begin{tabular}{|c||r|r|r|r|r||r|}
\hline \multicolumn{1}{|c||}{$r_{\text {甲 }}$} & \multicolumn{5}{c||}{ \# Real Solutions } & \multirow{2}{*}{ Total } \\
\cline { 2 - 6 } & 1 & 3 & 5 & 7 & 9 & \\
\hline \hline 4 & & & & & 7500 & 7500 \\
\hline 2 & 4995 & 13 & 4692 & & & 7500 \\
\hline 0 & & & 7500 & & & 7500 \\
\hline
\end{tabular}

Understanding these tables led Purbhoo [23] to study the number fixed points in a fiber of the Wronski map under the action of a cyclic or dihedral group. His Theorem 3.15 gives a formula for the number of real solutions to instances of Schubert problems $(\lambda, \lambda, \lambda, \lambda)$ with osculation type $r_{\lambda}=0$. The number of complex solutions to this problem is a particular set of Young tableaux, and Purbhoo's formula is the number of these Young tableaux that are fixed under an involution based on tableaux switching [2]. Example 3.16 of [23] gives the computation that this number is 4 for $\boxplus^{4}=8$ in $\operatorname{Gr}(3,7)$, as we saw in Table 9. Similarly, it is an exercise that this number is 5 for $\mathbb{Q}^{4}=9$ in $\operatorname{Gr}(4,8)$.

Purbhoo's result may be applied to Schubert problems in the family of Schubert problems $(a, 0)^{4}=a+1$ in $\operatorname{Gr}(2,2 a)$ when $r_{(a, 0)}=0$. As we see for the Schubert problem $\square^{4}=2$ of Table 8 and $\square^{4}=4$ in $\operatorname{Gr}(2,8)$ of Table 11, when there are no real points of osculation, these problems

TABLE 11. Frequency tables for $\square^{4}=4$ in $\operatorname{Gr}(2,8)$.

\begin{tabular}{|l||r|r|r||r|}
\hline \multicolumn{1}{|c||}{$r_{\text {r m }}$} & \multicolumn{3}{c||}{ \# Real Solutions } & \multirow{2}{*}{ Total } \\
\cline { 2 - 4 } & 0 & 2 & 4 & \\
\hline \hline 4 & & & 200000 & 200000 \\
\hline 2 & 32765 & 103284 & 63951 & 200000 \\
\hline 0 & & & 200000 & 200000 \\
\hline
\end{tabular}

appear to have $a+1$ real solutions. That is in fact always the case, as we now explain.

The solutions to $(a, 0)^{4}=a+1$ are enumerated by Young tableaux of shape $(2 a, 2 a)$ filled with $a$ copies of each of the numbers $1,2,3$, and 4 . Since the numbers 1 must fill the first $a$ positions in the first row and the numbers 4 must fill the last $a$ positions in the second row, the only choice is how many numbers 2 are in the first row. There are $a+1$ choices, so there are $a+1$ such tableaux. Here are the four tableaux for the problem 
$\square^{4}=4$.

\begin{tabular}{|l|l|l|l|l|l|l|l|l|l|l|l|l|l|l|l|l|l|l|}
\hline 1 & 1 & 1 & 2 & 2 & 2 \\
\hline 3 & 3 & 3 & 4 & 4 & 4 \\
\hline
\end{tabular}$\quad$\begin{tabular}{ll|l|l|l|l|l|l|l|}
\hline 1 & 1 & 1 & 2 & 2 & 3 \\
\hline 2 & 3 & 3 & 4 & 4 & 4 \\
\hline 2 & 2 & 1 & 2 & 3 & 3 \\
\hline 1 & 1 & 1 & 3 & 3 & 3 \\
\hline 2 & 2 & 2 & 4 & 4 & 4 \\
\hline
\end{tabular}

Purbhoo's switching involution switches the subtableaux consisting of the $1 \mathrm{~s}$ with that of the $2 \mathrm{~s}$, and that of the $3 \mathrm{~s}$ with that of the $4 \mathrm{~s}$. However, the properties of switching (see [2] or [23]) imply that every such tableaux is fixed under this involution, which implies that all solutions will be real for $(a, 0)^{4}=a+1$ with osculation type $r_{(a, 0)}=0$.

Despite this understanding for $r_{\lambda}=4$ and $r_{\lambda}=0$, we do not understand the possible numbers of real solutions when $r_{\lambda}=2$ for Schubert problems $(\lambda, \lambda, \lambda, \lambda)$.

\section{References}

[1] Monique Azar and Andrei Gabrielov, Some lower bounds in the B. and M. Shapiro conjecture for flag varieties, Discrete Comput. Geom. 46 (2011), no. 4, 636-659.

[2] Georgia Benkart, Frank Sottile, and Jeffrey Stroomer, Tableau switching: algorithms and applications, J. Combin. Theory Ser. A 76 (1996), no. 1, 11-43.

[3] W. Decker, G.-M. Greuel, G. Pfister, and H. Schönemann, Singular 3-1-6 - A computer algebra system for polynomial computations, 2012, http://www.singular.uni-kl.de.

[4] A. Martín del Campo and F. Sottile, Experimentation in the Schubert calculus, 2013, arXiv.org/1308.3284.

[5] D. Eisenbud and J. Harris, Divisors on general curves and cuspidal rational curves, Invent. Math. 74 (1983), no. 3, 371-418.

[6] A. Eremenko and A. Gabrielov, Degrees of real Wronski maps, Discrete Comput. Geom. 28 (2002), no. 3, 331-347.

[7] A. Eremenko and A. Gabrielov, Pole placement by static output feedback for generic linear systems, SIAM J. Control Optim. 41 (2002), no. 1, 303-312.

[8] , Rational functions with real critical points and the B. and M. Shapiro conjecture in real enumerative geometry, Ann. of Math. (2) 155 (2002), no. 1, 105-129.

[9] A. Eremenko, A. Gabrielov, M. Shapiro, and A. Vainshtein, Rational functions and real Schubert calculus, Proc. Amer. Math. Soc. 134 (2006), no. 4, 949-957 (electronic).

[10] Wm. Fulton, Young tableaux, London Mathematical Society Student Texts, vol. 35, Cambridge University Press, Cambridge, 1997.

[11] L. García-Puente, N. Hein, C. Hillar, A. Martín del Campo, J. Ruffo, F. Sottile, and Z. Teitler, The Secant Conjecture in the real Schubert calculus, Exp. Math. 21 (2012), no. 3, 252-265.

[12] J. Harris, Galois groups of enumerative problems, Duke Math. J. 46 (1979), 685724 .

[13] J. D. Hauenstein, N. Hein, A. Martín del Campo, and F. Sottile, Beyond the Shapiro Conjecture and Eremenko-Gabrielov lower bounds, 2010,

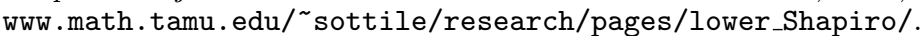

[14] N. Hein, C. Hillar, A. Martín del Campo, F. Sottile, and Z. Teitler, The Monotone Secant Conjecture in the real Schubert calculus, arXiv.org/1109.3436. 
[15] N. Hein and F. Sottile, Beyond the Shapiro Conjecture and Eremenko-Gabrielov lower bounds, 2013, http://www . math.tamu.edu/ secant/lowerBounds/lowerBounds.php.

[16] N. Hein, F. Sottile, and I. Zelenko, A congruence modulo four in the real Schubert calculus, 2012, arXiv.org/1211.7160.

[17] - A congruence modulo four for symmetric Schubert problems with real isotropic flags, 2013, in preparation.

[18] C. Hillar, L. García-Puente, A. Martín del Campo, J. Ruffo, Z. Teitler, S. L. Johnson, and F. Sottile, Experimentation at the frontiers of reality in Schubert calculus, Gems in Experimental Mathematics, Contemporary Mathematics, vol. 517, AMS, 2010, pp. 365-380.

[19] S. L. Kleiman, The transversality of a general translate, Compositio Math. 28 (1974), 287-297.

[20] E. Mukhin, V. Tarasov, and A. Varchenko, The B. and M. Shapiro Conjecture in real algebraic geometry and the Bethe ansatz, Ann. of Math. (2) 170 (2009), no. $2,863-881$.

[21] Schubert calculus and representations of the general linear group, J. Amer. Math. Soc. 22 (2009), no. 4, 909-940.

[22] K. Purbhoo, Reality and transversality for Schubert calculus in OG $(n, 2 n+1)$, Math. Res. Lett. 17 (2010), no. 6, 1041-1046.

[23] , Wronskians, cyclic group actions, and ribbon tableaux, Trans. Amer. Math. Soc. 365 (2013), no. 4, 1977-2030.

[24] J. Ruffo, Y. Sivan, E. Soprunova, and F. Sottile, Experimentation and conjectures in the real Schubert calculus for flag manifolds, Experiment. Math. 15 (2006), no. 2, 199-221.

[25] E. Soprunova and F. Sottile, Lower bounds for real solutions to sparse polynomial systems, Adv. Math. 204 (2006), no. 1, 116-151.

[26] F. Sottile, The special Schubert calculus is real, ERA of the AMS 5 (1999), 35-39.

[27] Real Schubert calculus: Polynomial systems and a conjecture of Shapiro and Shapiro, Experiment. Math. 9 (2000), no. 2, 161-182.

[28] , Frontiers of reality in Schubert Calculus, Bull. Amer. Math. Soc. 47 (2010), no. 1, 31-71.

[29] , Real solutions to equations from geometry, University Lecture Series, vol. 57, American Mathematical Society, Providence, RI, 2011.

[30] Enrique A. Tobis, rootsur_lib A SINGULAR 3-1-6 library for counting the number of real roots of univariate polynomial, 2012, http://www.singular.uni-kl.de.

[31] R. Vakil, Schubert induction, Ann. of Math. (2) 164 (2006), no. 2, 489-512.

[32] Jan Verschelde, Numerical evidence for a conjecture in real algebraic geometry, Experiment. Math. 9 (2000), no. 2, 183-196. 